\title{
Erratum: Hamiltonian for the inclusion of spin effects in long-range Rydberg molecules [Phys. Rev. A 95, 042515 (2017)]
}

\author{
Matthew T. Eiles and Chris H. Greene
}

(Received 12 June 2018; published 5 July 2018)

DOI: 10.1103/PhysRevA.98.019901

There are several typographical errors in the "multipole moments" section along with some poor and confusing notational conventions. The changes below rectify these issues. In particular, several times the letter $L$ was used rather than the letter $k$, or the letter $k$ was used with multiple meanings. Making the appropriate replacements frees $L$ to replace $L^{\prime}$, which improves the legibility of this notation. We additionally removed two extraneous expressions [Eqs. (25) and (26) in the original article] involving reduced matrix elements, which are unrelated to the final result and thus introduced additional confusion due to their dependence on the convention of the Wigner-Eckart theorem.

These replacements are as follows:

Equation (22) should be replaced with

$$
T_{q}^{k}=-r^{k} C_{k q}(\hat{r}), \quad C_{k q}(\hat{r})=\sqrt{\frac{4 \pi}{2 k+1}} Y_{k q}(\hat{r}) .
$$

Equation (23) should be replaced by

$$
\Psi_{L M_{L}}(\vec{R}, \vec{r})=\frac{\sum_{l} Q_{L M_{L}}^{n l}(R) Y_{l M_{L}}(\hat{r}) r^{-1} f_{n l}(r)}{\sqrt{\mathcal{N}_{n L M}(R)}},
$$

where $\mathcal{N}_{n L M}(R)=\sum_{l}\left|Q_{L M_{L}}^{n l}(R)\right|^{2}$. Equation (24) should be replaced by

$$
\begin{aligned}
\left\langle T_{q}^{k}\right\rangle & =\left\langle\Psi_{L M}\left|T_{q}^{k}\right| \Psi_{L M}\right\rangle \\
& =\sum_{l, l^{\prime}} \frac{Q_{L M}^{n l}(R) Q_{L M}^{n l^{\prime}}(R)}{\mathcal{N}_{n L M}(R)}\left\langle n l M\left|T_{q}^{k}\right| n l^{\prime} M\right\rangle .
\end{aligned}
$$

The text and equations between Eq. (24) and [including Eq. (28)] should be replaced with:

The matrix element separates into a radial integral $R_{n l}^{n^{\prime} l^{\prime}}(k)=\int d r f_{n l}(r) r^{k} f_{n^{\prime} l^{\prime}}(r)$ multiplying an angular integral which is evaluated with standard techniques to obtain the result,

$$
\left\langle T_{q}^{k}\right\rangle=\sum_{l, l^{\prime}} \frac{Q_{L M}^{n l}(R) Q_{L M}^{n l^{\prime}}(R)}{\mathcal{N}_{n L M}(R)} R_{n l}^{n l^{\prime}}(k) C_{l^{\prime} M, k q}^{l M}(-1)^{k-l^{\prime}} \sqrt{\left(2 l^{\prime}+1\right)}\left(\begin{array}{ccc}
l & l^{\prime} & k \\
0 & 0 & 0
\end{array}\right) .
$$

The Clebsch-Gordan coefficient causes any term with $M^{\prime} \neq 0$ to vanish, reflecting the cylindrical symmetry. The $k=1$ moments agree exactly with Ref. [18]. These multipole moments scale in size as $n^{2 k}$ and are displayed in Fig. 8 up to the octupole moments. Within the full spin model, the multipole moments are derived similarly but using the numerically calculated eigenstates $|s\rangle=\sum_{\kappa} a_{s \kappa}|\kappa\rangle$, where $|s\rangle$ is an electronic eigenstate, $\kappa$ is a composite quantum number $\kappa=\left\{n\left(l s_{1}\right) j m_{j} m_{2} m_{i}\right\}$, and $a_{s \kappa}$ is the eigenvector corresponding to the $s$ th eigenstate. The multipole moments are then

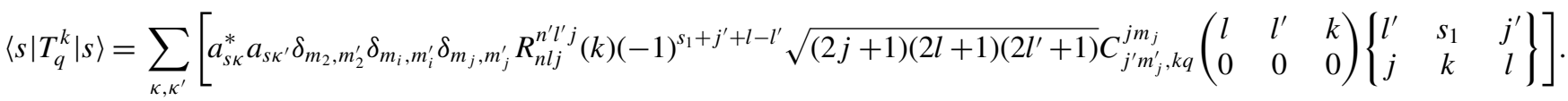

These typographical errors or confusing conventions do not affect any other part of the paper or the calculations displayed in Figs. 8 and 9. 